\title{
Organ Donation and Utilization in the United States, 1996-2005
}

\author{
J. D. Punch ${ }^{a}$ * , D. H. Hayes ${ }^{b}$, F. B. LaPorte ${ }^{c}$, \\ V. McBride ${ }^{d}$ and M. S. Seely ${ }^{e}$ \\ a Department of Surgery, University of Michigan, Ann \\ Arbor, Michigan, USA \\ b LifeShare of the Carolinas, Charlotte, North Carolina, \\ USA \\ 'Scientific Registry of Transplant Recipients (SRTR), Arbor \\ Research Collaborative for Health, Ann Arbor, Michigan, \\ USA \\ dDepartment of Health and Human Services, Health \\ Resources and Services Administration, Rockville, \\ Maryland, USA \\ e Pacific Northwest Transplant Bank, Oregon Health \& \\ Science University, Portland, Oregon, USA \\ * Corresponding author: Jeffrey D. Punch, \\ jpunch@med.umich.edu
}

\begin{abstract}
The success of clinical transplantation as a therapy for end-stage organ failure is limited by the availability of suitable organs for transplant. This article discusses continued efforts by the transplant community to collaboratively improve the organ supply. There were 7593 deceased organ donors in 2005 . This represents an all-time high and a 6\% increase over 2004. Increases were noted in deceased organ donation of all types of organs; notable is the increase in lung donation, which occurred in $17 \%$ of all deceased donors. The percentage of deceased donations that occurred following cardiac death has also reached a new high at $7 \%$. The number of living donors decreased by $2 \%$, from 7003 in 2004 to 6895 in 2005 . This article discusses the continued efforts of the Organ Donation Breakthrough Collaborative and the Organ Transplantation Breakthrough Collaborative to support organ recovery and use and to encourage the expectation that for every deceased donor, all organs will be placed and transplanted.
\end{abstract}

Key words: Deceased donors, donors after cardiac death, living donors, Organ Donation Breakthrough Collaborative, OPTN, SRTR

\section{Introduction}

The obstacle that most frequently prevents patients from benefiting from organ transplantation continues to be the availability of suitable organs. Despite considerable efforts aimed at public awareness of the need for organ donation, the supply of organs has simply not kept up with demand.
For many years, it seemed that the transplant community accepted this situation as the unfortunate, but inevitable, consequence of the increased demand caused by the success of transplantation, coupled with an intrinsically limited supply of organs. However, in the past 3 years, the gradual, decade-long increase in organ donation appears to have accelerated perceptibly.

There are many possible explanations for the foregoing changes. Greater scrutiny is now being placed on organ utilization, forcing transplant programs to carefully examine organ acceptance policy and available staffing. Greater interest in the use of expanded criteria donors (ECD) has also developed. National consensus conferences have examined this issue, as well as questions about how the transplant community can increase the utilization of organs from donors following cardiac death (DCD). State-sponsored organ donor registries are becoming established in many parts of the country and now actually may be affecting overall donation. Notable changes in organ allocation policy have occurred that may be improving organ utilization, including a revised lung allocation system based on a severity of illness scale. Finally, concerted efforts, guided by the federal government, are being made to disseminate best practices within the transplant community in hopes of increasing the supply of deceased donor organs.

In 2003, with guidance from the leadership of Health and Human Services (HHS), the Health Resources and Services Administration (HRSA) set out to alter the disappointing trend of the previous decade by engaging the transplant community in an effort called the Organ Donation Breakthrough Collaborative. This article will review and analyze trends in organ donation in the United States over the past 10 years, including the last 31 months since the first Collaborative meeting in September 2003. It also will discuss the progress of the Collaborative toward changing both attitudes and practices regarding donation.

\section{Trends in Deceased Organ Donation}

A marked increase in deceased donation has occurred since the beginning of the Collaborative. In the 6 years prior to its development (1996-2002), the number of deceased organ donors increased from 5418 to 6190, an average of $2 \%$ per year. In comparison, the number of deceased organ donors has increased an average of $8 \%$ per year, from 6457 in 2003 to 7593 in 2005 . Notably, the number 


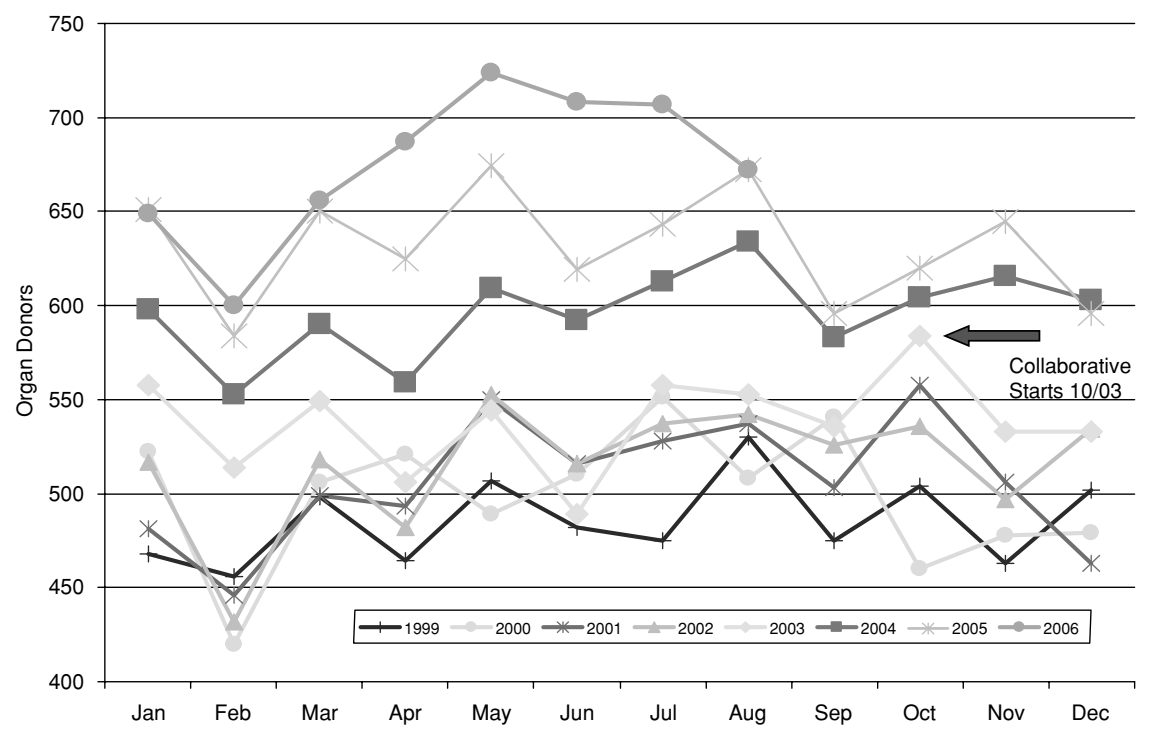

Source: Organ Donation Breakthrough Collaborative.

Figure 1: Month-by-month increases in number of organ donors.

of organs donated each month has exceeded the number in the corresponding month from the previous year for 30 months in a row (Figure 1). Given the numerous feasible explanations for the increase, it is not possible to know what portion, if any, of the increased donation has been the result of the Collaborative. Nevertheless, the association is striking, given that the accelerated rate of increasing donation correlates in time with the beginning of the Collaborative era.

Total organ donation (deceased plus living) has steadily increased over the past 10 years, from 9208 organ donors in 1996 to 14488 donors in 2005. The percentage change from year to year has varied from $1 \%$ to $10 \%$, with the largest overall increase seen between 1999 and 2000, when the number of living donors increased by $18 \%$, from 5037 to 5933 . The number of donors in 2004 increased $6 \%$ from 2003 , primarily because of an $11 \%$ increase in deceased donors. The number of total donors increased $2 \%$ from 2004 to 2005, despite a $2 \%$ decline in the number of living donors; the latter decline can be said to have occurred because there was a $6 \%$ rise in deceased donors.

The increase in organ donation is observed in all organs except heart, where donation rates have slowly decreased from 1996 to 2004 and then increased by nearly 6\% from 2004 to 2005. The overall increase in donation rates is largely driven by kidney and liver donation, where the 10year increases in organ donors (living and deceased donors combined) were 4549 and 2486, respectively. It is worth noting that the relative increase in lung donation exceeded $18 \%$ from 2004 to 2005.

Over the past 5 years, living organ donation has remained relatively constant, while the number of deceased donors has increased. In 2005, this trend is again observed across all organs except for intestine, where the number of living donors is small but similar to 2004. From 2004 to 2005, for the first time, the number of living donors decreased by $2 \%$ (7003 to 6895).

\section{Deceased donors}

The number of deceased donors has increased almost $40 \%$ from 1996. As mentioned earlier, most of this increase is seen from 2000 to 2005. Prior to 2000, the deceased donation rate remained relatively constant. In each calendar month of 2005 the number of organ donors was greater than in the corresponding month in the previous year. All organs showed an increase; the greatest increase was $20 \%$ in lung donation from deceased donors and the smallest increase was in pancreas donation (1\%). The 6\% increase in donated hearts (from 2096 to 2220) represents the first time in 10 years that heart donation has increased compared with the previous year. The overall increase in donor organs resulted from an increase of $8 \%$ in the number of organs from donors aged 18 years and over (6693 from 6175), while the number of organs from donors less than 18 years fell by $8 \%$ (from 975 to 900). A slight but consistent increase in the proportion of donors over 65 years is noted, from $9.7 \%$ in 2000 to $10.4 \%$ in 2005. The distribution of causes of death among organ donors was virtually unchanged compared with 2004. Similarly, the distribution of donor ethnicity has remained relatively unchanged, with the exception of a subtle increase in the percentage of African Americans donors, from 14\% to $15 \%$, and a corresponding decrease in the percentage of white donors, from $70 \%$ to $68 \%$. These data suggest that efforts to improve organ donation rates have been effective across demographic groups and in multiple clinical organ donation settings. 
The number of donors who died of head trauma has decreased markedly over the past 10 years. The proportion of donors with head trauma in 1996 was $45 \%$, but in 2005, the proportion dropped to $38 \%$. Again, most of the change seems to have taken place over the last 5 years, along with an increase in anoxia (10-15\%). Deaths due to motor vehicle accidents have also declined, from $25 \%$ in 1996 to $20 \%$ in 2006 . The majority of donors are classified into 'other' circumstances of death; this category has increased from $49 \%$ in 1996 to $57 \%$ in 2005 .

While the total count of deceased donors has improved over time, the relative increase has not been the same for all donor types. DCD has shown the greatest percentage increase over the past 10 years. In 1996, organs were recovered from 71 DCD (1\%); however, by 2005, that number increased more than six times to 561 donors (7\%). ECD have more than doubled, from 990 in 1996 to 2027 in 2005. The definition for ECD are donors aged $\geq 60$ years or donors aged 50-59 years with at least two of the following conditions: cerebrovascular accident as cause of death, serum creatinine $>1.5 \mathrm{mg} / \mathrm{dL}$ or a history of hypertension. Recipients of ECD kidneys have a relative risk of graft loss greater than 1.70 compared with kidneys from a reference group of donors aged 10-39 years without any of the other three conditions (1). Conversely, the number of standard criteria donors (SCD) has increased only very slightly. SCD are defined as donors after brain death (DBD), whose kidneys do not fit the description for ECD. The number of SCD in 2005 is $0.5 \%$ higher than that of 2004, and the largest increase noted in the past 10 years for SCD is $8 \%$ (2003-2004). These data show that the increase seen in deceased donors over the past 10 years is almost entirely due to large increases in DCD and ECD. The fraction of the deceased donor population coming from SCD has been decreasing each year with the simultaneous increase in DCD and ECD (Figure 2). However, SCD still represented more than $67 \%$ of all donors in 2005.

Despite extensive efforts on the part of the Organ Donation Breakthrough Collaborative to increase conversion rates, the overall number of organs recovered per donor (ORPD) and organs transplanted per donor (OTPD) remained stable in 2005 at 3.53 and 3.06, respectively. (The conversion rate, also called the donation rate, is calculated as the number of actual donors aged 70 years or younger from whom at least one organ is recovered for the purpose of transplant divided by the total number of eligible deaths, i.e. donors also aged 70 years or younger that are brain dead and not diagnosed with exclusionary medical conditions.) The explanation for the stability in the number of organs recovered and transplanted per donor appears to be the increasing percentage of donors that are ECD and DCD, which are associated with a lower number of OTPD. In 2005, SCD decreased to $67 \%$ of donors from $71 \%$, while ECD increased from $24 \%$ to $27 \%$ and DCD increased from $4 \%$ to $6 \%$. Since the OTPD from ECD and DCD is less

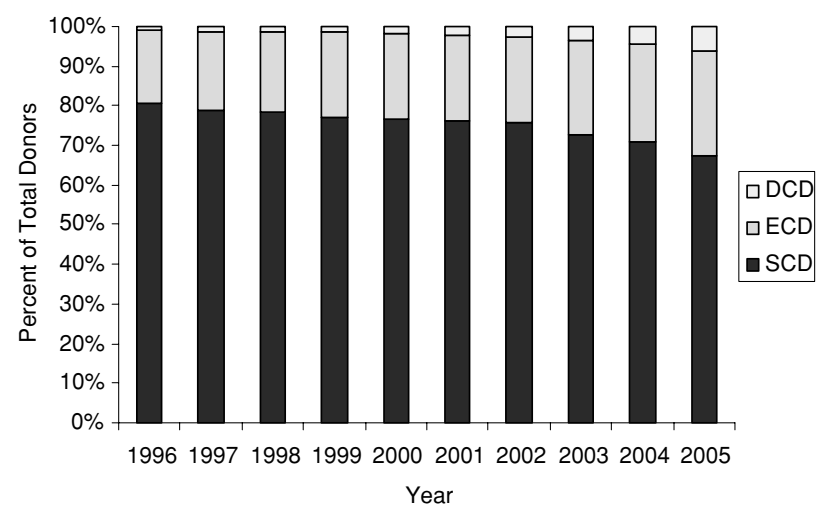

Source: 2006 OPTN/SRTR Annual Report, Table 2.12.

Figure 2: Deceased donor population, by donor type and year, 1996-2005.

than from SCD and because the OTPD increased by more than $2 \%$ for SCD (as it did for ECD), the decrease in the percentage of SCD is largely counterbalanced in the distribution of organs from these donors. The number of OTPD from DCD decreased by $2 \%$, from 2.20 to 2.16 . This may have been because support for the use of DCD kidneys is spreading more quickly than interest in other organs from DCD donors. Therefore, as the number of DCD rise, the use of extra-renal organs from DCD initially may lag.

Deceased kidney donation: In 2005, there were 9342 kidneys recovered from SCD, 3102 kidneys recovered from ECD and 869 kidneys recovered from DCD. These numbers all reflect increases from 1996 (14\%, 78\% and 697\%, respectively); however, the numbers of ORPD and OTPD have not improved. For SCD and DCD kidney donors, the ORPD has remained about the same, but ECD kidney donors have dropped from 1.76 in 1996 to 1.53 ORPD in 2005. OTPD has dropped for all organ types: from 1.73 to 1.68 for SCD donors, 1.16 to 0.9 for ECD donors and 1.63 to 1.55 for DCD donors.

Deceased pancreas donation: There have been increases in pancreata recovered from both SCD and DCD, though the vast majority of pancreata are recovered from the former. Of the 2033 pancreata recovered in 2005, 1964 were recovered from DBD. Over the past 10 years, the number of pancreas grafts transplanted rose gradually, from 1014 in 1996 to 1459 in 2002, from which point the number has remained relatively stable. The number of pancreata transplanted in 2005 declined slightly compared with 2004, from 1504 to 1453.

Deceased liver donation: Of the 6690 livers recovered in 2005, 388 were recovered from DCD. This is a dramatic increase, as described above. The number of SCD livers that were recovered and transplanted has remained about the same over the past 10 years; however, the number of livers recovered and transplanted from DCD gradually has improved. In 1996, the average number of livers recovered per DCD was 0.29 , but by 2005 , the 
average number of livers recovered per DCD was 0.72 . Similarly, among DCD, the number of livers that were actually transplanted per donor rose from 0.21 to 0.52 .

Deceased heart donation: As previously reported, the number of deceased heart donors has declined over the past 10 years. In addition, the number of hearts recovered from those donors that are consented is decreasing as well. In 2005, only 2220 hearts were recovered from 7593 donors compared with 2462 hearts recovered from 5418 donors in 1996. Hearts recovered from donors over the past 10 years have almost exclusively been from SCD; five instances are reported where a DCD heart was recovered for transplant. Of the hearts recovered from DBD, the ORPD and OTPD have gradually declined, from 0.46 to 0.31 and 0.44 to 0.31 , respectively.

Deceased intestine donation: The number of intestines recovered from deceased donors has steadily risen, from 48 in 1996 to 184 in 2005 . In the past 10 years, only one intestine has been recovered from a donor following cardiac death and it was not transplanted. Of the intestines recovered from SCD, a slight increase (approximately $2 \%$ ) is observed in recovery and transplantation.

Deceased lung donation: The number of lung donors has increased from 1401 in 1996 to 2374 in 2005 . The majority of these were also recovered from DBD; in 2005, only 12 lungs were recovered from DCD. Between 1996 and 2005, the ORPD and OTPD have gradually increased from DBD that provided lungs, from 0.26 to 0.33 and 0.23 to 0.32 , respectively. Although the numbers are small, there is an increase in the number of lungs recovered and transplanted from DCD, from 2001 to 2005.

\section{Deceased organ distribution}

Changes in organ allocation and distribution policy have had negligible effects on the distribution of organs in 2005, as measured by whether the organs were transplanted locally or shared compared with 2004, with the exception of livers. The percentage of organs used locally varied widely by organ type. Of kidneys transplanted, $71 \%$ were used locally; $61 \%$ of transplanted hearts were transplanted locally and $52 \%$ of lungs. All of these percentages remained stable in 2005 relative to 2004 . In contrast, the percentage of livers transplanted locally decreased from $69 \%$ in 2004 to $66 \%$ in 2005 . A significant reason for this trend could be the effect of an allocation policy enacted by the OPTN in 2005 directing livers regionally if a local candidate with a Model for End-stage Liver Disease (MELD) score of at least 15 is not available.

\section{Expanded criteria donation}

The number of donated kidneys that met the definition of ECD increased by 16\%, from 1340 in 2004 to 1562 in 2005. This change represented almost $60 \%$ of the overall increase in organs donated, despite the fact that ECD still represent only about one-fourth of all deceased donors. Kidneys from this important source of organs are allocated to those also listed as ECD candidates, using an algorithm based purely on waiting time rather than on HLA match. A component of the increase that has been observed in ECD kidneys relative to that observed with non-ECD kidneys appears to be due to the refinement in kidney allocation policy that was aimed at reducing the number of kidneys discarded because of prolonged ischemic times (2). Overall, the ECD program appears to be a success. The survival benefit of these kidneys has been shown to be substantial for candidates who are older than 40 years in OPOs with long waiting times; in OPOs with shorter waiting times ECD benefit is seen only for patients with diabetes (3). The expedited allocation process has also been reported to reduce cold ischemia time and delayed graft function in some areas (4).

\section{Donation after cardiac death}

The most remarkable increase in any category of organ donation in 2005 has been the $43 \%$ increase in DCD, from 391 in 2004 to 561 in 2005 . While DCD still represent only $7 \%$ of all organ donors, the dramatic rate of increase suggests that an untapped potential source of organs may be available. Table 1 shows the characteristics of DCD donors in 2005. Historically, the rate of DCD organ recovery by OPOs and the use of DCD organs by transplant programs have shown great variation. To address this shortcoming, a national consensus conference on DCD was held in Philadelphia in the spring of 2005 (5). The group established standardized definitions and principles in many areas pertaining to DCD. The group also affirmed the position of the Institute of Medicine that this type of donation is both an ethical practice and an important source of lifesaving organs. The Organ Donation Breakthrough Collaborative also maintained a strong focus on increasing the number of DCD in each DSA.

DCD kidney donors: Of the 561 DCD in 2005, 530 (94\%) were kidney donors. In 2005, 93 of the 530 DCD for kidney also met the ECD definition (18\%). In 2004, a similar fraction of the kidney DCD met the ECD definition (63 of $371 ; 17 \%)$. These data suggest that the recent increase in the number of organs from DCD has not been due to the relaxation of acceptance standards for DCD organs, but to the expansion of DCD recovery throughout the donor pool.

DCD pancreas donors: The number of pancreata recovered from DCD continues to increase rapidly but still only represents $4 \%$ of the overall pancreas donor pool. In 2005 , there were 72 DCD for pancreas, an increase of $47 \%$ from the 49 pancreas donors in 2004 . The percentage of DCD that were pancreas donors in 2005 was $15 \%$, unchanged from 2004.

DCD liver donors: The number of DCD in which the liver was recovered increased by 60\%, from 242 in 2004 to 388 
Table 1: Characteristics of DCD in 2005

\begin{tabular}{|c|c|c|c|}
\hline & All DCD & Controlled & Uncontrolled \\
\hline Total number of donors & 473 & 458 & 15 \\
\hline Mean donor age (years) & 39.7 & 39.8 & 34.5 \\
\hline $\begin{array}{l}\text { Mean number of organs } \\
\text { recovered }\end{array}$ & 2.8 & 2.8 & 3.1 \\
\hline $\begin{array}{l}\text { Mean number of organs } \\
\text { transplanted }\end{array}$ & 2.3 & 2.3 & 2.7 \\
\hline $\begin{array}{l}\text { Organs transplanted } \\
\text { locally, regionally, } \\
\text { nationally }{ }^{1}\end{array}$ & $\begin{array}{c}(819,109 \\
166)\end{array}$ & $\begin{array}{c}(788,105 \\
160)\end{array}$ & $(31,4,6)$ \\
\hline
\end{tabular}

${ }^{1}$ Local, regional and national counts are mutually exclusive. Source: SRTR analysis, November 2006.

in 2005. Livers from DCD now represent $6 \%$ of the overall liver donor pool. The percentage of DCD that were liver donors in 2005 was $72 \%$, up from $64 \%$ in 2004. Increased use of livers from DCD may be due in part to single-center evidence that acceptable patient survival is attainable with selected DCD livers (6). However, at the national level the use of DCD livers was found to be associated with a significantly higher risk of graft failure versus DBD livers when studied by multivariable analysis (7). Thus, there is a need for continued efforts aimed at improving outcomes for recipients of DCD livers.

DCD lung donors: The use of lungs from $D C D$ continues to be rare. Lungs were used from seven DCD in 2005, representing less than $1 \%$ of all deceased donor lungs. However, activity may expand in the future as DCD in general become more commonplace. It also is likely to increase as encouraging preliminary experience, primarily at the University of Wisconsin, showing excellent success rates for lungs procured from DCD becomes more widely known (8).

\section{Implementing the Breakthrough Collaborative Improvement Strategy}

The principles and goals of the Organ Donation Breakthrough Collaborative have been described previously (9). Hospitals were encouraged to send senior leadership members and front-line staff members with the authority to implement change to meetings of the Organ Donation Breakthrough Collaborative. Organ procurement organizations (OPOs) were asked to partner with their hospital colleagues in a systematic way to implement specific, highleverage changes (Table 2). A set of initial expectations was defined as key to achieving the $75 \%$ donation rate goal in every hospital in the United States with three or more eligible donors per year. A 'first-things-first' strategy was outlined that hinged on the identification of organ donation advocates from the hospital staff, both physician and nonphysician, to lead donation efforts and to team with an inhouse OPO coordinator, who, while employed by the OPO, is based primarily at the hospital or health system. These
Table 2: High leverage changes for OPOs

Advocate organ donation as the mission

Involve senior leadership to get results

Deploy a self-organizing OPO/hospital team

Practice early referral, rapid response

Master effective requesting

Implement donation after cardiac death

Source: Organ Donation Breakthrough Collaborative.

teams were asked to develop processes for conducting real-time death record reviews and to focus on analysis of current hospital data and action plans. The routine use of clinical triggers, team huddles, effective requestors and after-action reviews was also stressed.

In 2005, the Organ Donation Breakthrough Collaborative expanded beyond the original 225 participating hospitals to the approximately 850 hospitals in the United States that account for $90 \%$ of the nation's eligible donors. The benefits of focusing on hospitals with a sizable number of eligible donors can be easily understood by considering the 287 hospitals with 12 or more eligible donors per year (Figure 3). If the conversion rate for each hospital was raised from its observed 2005 performance level to the $75 \%$ goal, an additional 1053 organ donors would result, an increase of roughly $14 \%$. This projection does not include any other additional sources such as DCD or donors over the age of 70 years, since these donors are not included in the conversion rate calculation. Considering that the number of hospitals with 12 or more eligible donors within each donation service area (DSA) ranges from 1 to a maximum of 15 , it appears that the $75 \%$ goal in each hospital is achievable, even in the largest DSAs.

These efforts being made through the Collaborative are intended to identify barriers to conversion and to encourage rapid adoption of measures in order to positively influence conversion rates. It can be noted that the national donor conversion rate has edged up steadily from $52 \%$ in 2003 to $57 \%$ in 2004 and $58 \%$ in 2005 (Table 3).

The Collaborative proved effective at attracting the attention of donor hospital executives, perhaps, in part, because of the pending standards regarding organ donation that were established by the Joint Commission for Accreditation of Healthcare Organizations (10). These standards send the strong message that a highly performing organ and tissue donation program is an expectation of every accredited hospital.

The Collaborative's strategy proved effective at generating interest beyond the national gatherings. Streamlined versions of the national learning sessions, known as 'minicollaboratives', began appearing in many individual DSAs as a means of disseminating both donation best practices and the philosophy of partnership and joint accountability between OPOs and hospitals as a means to improve donation rates. OPOs and hospitals together developed 


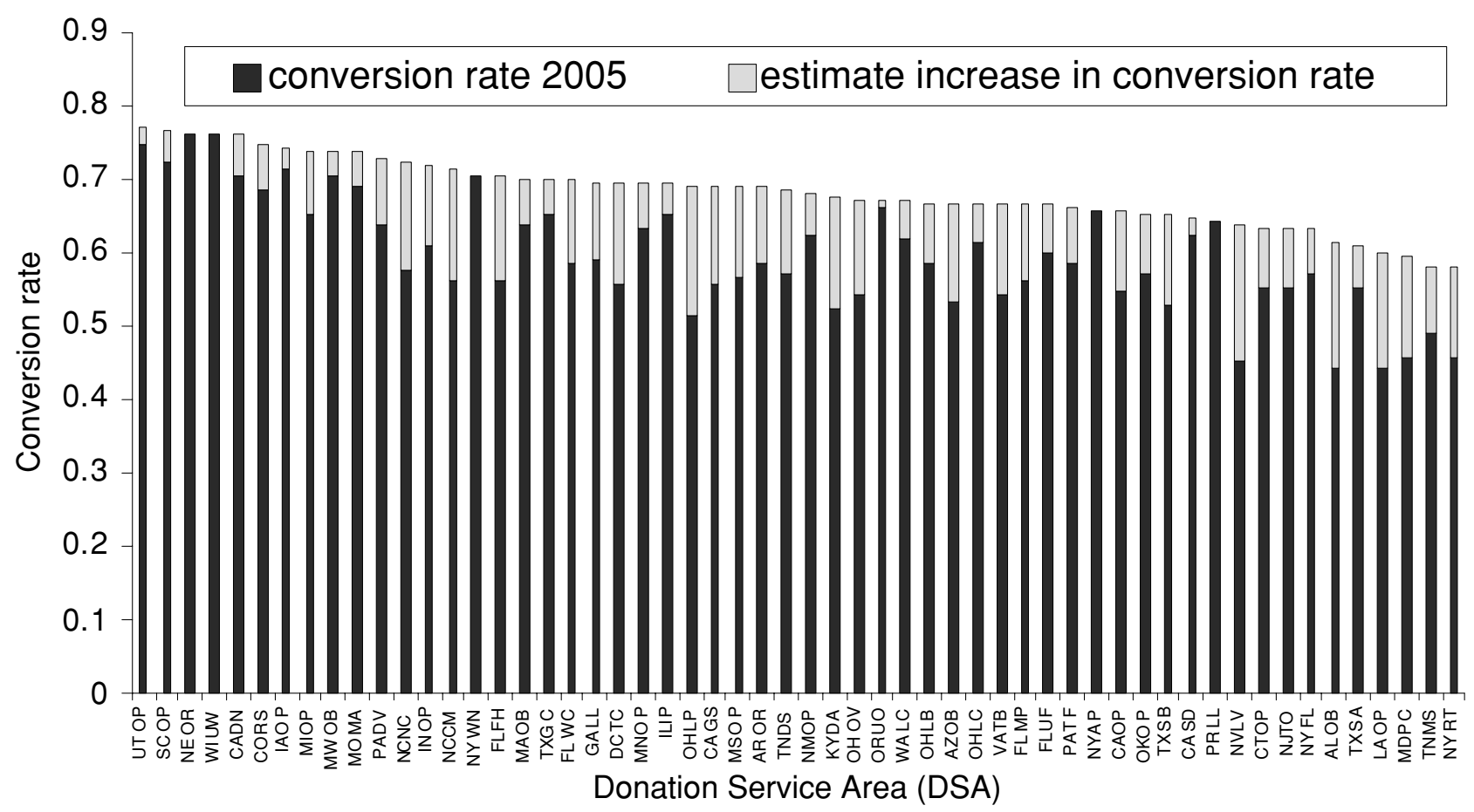

* Large hospitals defined as $\geq 12$ eligible deaths

Source: Organ Donation Breakthrough Collaborative.

Figure 3: 2005 Conversion rate and estimated conversion rate if conversion rate was $\mathbf{7 5} \%$ for all large hospitals.

'dashboards' composed of succinct graphics and text to illustrate whether organizations were meeting established targets such as donor referral rate, appropriate request rate and consent rate. Many hospitals adopted these targets as components of their comprehensive quality improvement programs and used the dashboards as a means to track progress and communicate results. OPOs and hospitals began collaborating with state hospital associations to foster ongoing partnerships to meet and exceed the $75 \%$ goal statewide.

OPOs and hospitals that achieve the $75 \%$ donation rate target are recognized by HHS with an Organ Donation Medal of Honor. In 2004, 184 hospitals maintained $75 \%$ donation rates for a consecutive 12 -month period and merited this distinction (11). Using the same criteria in 2005, nearly 270 hospitals sustained the $75 \%$ donation goal for 12 months, an increase of $44 \%$.

Table 3: Reported eligible deaths, actual donors and conversion rate, 2002-2005

\begin{tabular}{lllll}
\hline & $\mathbf{2 0 0 2}$ & $\mathbf{2 0 0 3}$ & $\mathbf{2 0 0 4}$ & $\mathbf{2 0 0 5}$ \\
\hline Actual donors & 5746 & 5912 & 6447 & 6666 \\
Eligible deaths & 11503 & 11375 & 11365 & 11412 \\
Conversion rate & $50.0 \%$ & $52.0 \%$ & $56.7 \%$ & $58.4 \%$ \\
\hline
\end{tabular}

Source: SRTR Analysis, August 2006.

Conversion rate $=$ actual donors $\leq 70$ years/reported eligible deaths.

\section{OPO Redesign}

These sustained monthly donation records prompted OPOs to consider their own design and operations. This internal reflection was driven by two forces: the need to create capacity to handle significant increases in donor activity and the need to systematically and robustly apply quality improvement methods to sustain and build on this growth. To do this, OPOs have begun a process of redesign.

Seven drivers of organizational change anchored the OPO redesign efforts (Table 4). OPOs have been encouraged to examine their performance in each area and to work with their colleagues to learn how successful organizations are designed and function. Essential to the redesign process is securing the interest of representatives from all levels of the organization, from the board of directors and executive director through the middle managers and front-line staff. Using the same improvement methodology integral to the Collaborative, OPO redesign teams have begun testing and implementing changes that should enable them to achieve DSA-wide conversion rates of $75 \%$ and to be better prepared to meet the challenges of the new Organ Transplantation Breakthrough Collaborative.

Only $21 \%$ of OPOs experienced a decrease in actual donors in 2005, compared with $31 \%$ in 2004. There was an increase in the number of OPOs whose pattern of recovery involved 2 consecutive years of growth for 2004 and 
Table 4: Seven drivers of organizational change

\author{
Culture/vision \\ Business case/pre-emptive financing \\ Staffing/structure \\ Goal setting/measurement \\ Quality/process improvement \\ Collaboration/relationships \\ System/methods
}

Source: Organ Donation Breakthrough Collaborative.

2005 (Table 5). No OPO experienced a 2-year consecutive decline.

\section{Organ Transplantation Breakthrough Collaborative}

A key observation during the Organ Donation Breakthrough Collaborative was the level of variability among DSAs in the rate of OTPD, ranging in 2003 from 2.4 to 4.3 for SCD (Figure 4).

Additionally, a notable decrease in OTPD among SCD coinciding with the start of the Organ Donation Breakthrough Collaborative (Figure 5) underscored the need to identify and disseminate practices that would bolster organ recovery and transplantation rates that were dropping under the stress of increasing donor activity. With this in mind, the Organ Transplantation Breakthrough Collaborative was established in October 26, 2005, with the goal of raising the national OTPD rate from 3.06 to 3.75 . This goal has been further refined into sub-goals based on donor type: 4.3 organs per SCD, 2.75 organs per DCD and 2.5 organs per ECD. An additional goal challenges each DSA to increase the number of DCD until they represent at least $10 \%$ of the DSA's total donors without negatively affecting the number of DBD.

The Transplantation Collaborative is based on strategies identified as essential to increasing OTPD. OPOs are encouraged to approach every organ donor with the expectation that all organs will be placed and transplanted ('every donor, every organ, every time'). Transplant Collaborative teams are encouraged to build upon the partnerships that have developed among OPO staff and donor hospital intensivists and ICU staff during the Donation Collaborative. This existing network should help advance effective organ donor management practices and expand and im-

Table 5: Growth and decline in OPO recovery 2004-2005

\begin{tabular}{lll}
\hline $\begin{array}{l}\text { Pattern of } \\
\text { recovery }\end{array}$ & $\begin{array}{l}\text { Number } \\
\text { of OPOs }\end{array}$ & $\begin{array}{l}\text { Percentage } \\
\text { of total }\end{array}$ \\
\hline Two consecutive years of growth & 25 & $43 \%$ \\
Two consecutive years of decline & 0 & $0 \%$ \\
Growth in 2004 and decline in 2005 & 14 & $24 \%$ \\
Decline in 2004 and growth in 2005 & 17 & $29 \%$ \\
Growth in 2004 and no change in 2005 & 2 & $4 \%$ \\
No change in 2004 and decline in 2005 & 0 & $0 \%$ \\
No change in 2004 and growth in 2005 & 0 & $0 \%$ \\
Total & 58 & $100 \%$
\end{tabular}

Source: OPTN Analysis, March 2006. plement proven DCD identification and procurement protocols. Organ Transplantation Breakthrough Collaborative teams are now being joined by transplant physicians, surgeons, coordinators and administrators, who can advocate for enhanced donor evaluation protocols, assess the effectiveness of organ offer acceptance or refusal policies and evaluate the appropriateness of those decisions.

The average number of organs transplanted per month during the first year of the Organ Donation Breakthrough Collaborative (October 1, 2003, to September 30, 2004) was 1782 , an increase of $6 \%$ over the previous 12 -month period. With the Collaborative's ongoing emphasis on 'every donor, every organ, every time', the number of monthly transplants rose an additional $8 \%$ in the next 12 -month period (October 1, 2004, to September 30, 2005) (Figure 6). This performance level has been maintained during the first 5 months of the Transplantation Collaborative (beginning October 26, 2005) and is expected to increase in response to the intensive focus of OPOs, donor hospitals and transplant programs.

\section{Recent Developments in Deceased Donation}

\section{Definition of a potential organ donor}

The standard definition of a potential organ donor has been under discussion over the last 5 years, with some resolution and agreement among the Association of Organ Procurement Organizations (AOPO), the OPTN, HRSA and the Centers for Medicare and Medicaid Services (CMS). The OPO community came to consensus in June 2005 and made comment to CMS, whose definition of 'organ donation potential' is reflected in section 486.302 of the Final Rule regulating OPOs (12). AOPO expressed concern that the CMS definition differed from that used by the OPTN for reporting purposes. This lack of a uniform definition likely would cause confusion and lead to inaccuracies, making meaningful comparisons across DSAs difficult. In response, AOPO recommended a definition for 'organ donor potential' that makes use of exclusionary criteria (Table 6).

This definition was subsequently approved by the OPTN Board of Directors and HRSA for reporting purposes, and CMS adopted the definition in its June 2006 publication of the Final Rule for OPO Conditions of Coverage. The benefit will be consistency in reporting, which in turn will support clearer and enhanced comparisons. However, it should be noted that eligible donors are self-reported by hospitals and are thus unconfirmed. While CMS accepted this as a performance measure for OPOs, this inherent flaw in the data collection method must be considered when interpreting conversion rates.

\section{Donor registries}

The 1998 Uniform Anatomical Gift Act set the stage for the development of donor registries that would feature the simplicity of donor cards and electronic formats, allowing 
Punch et al.

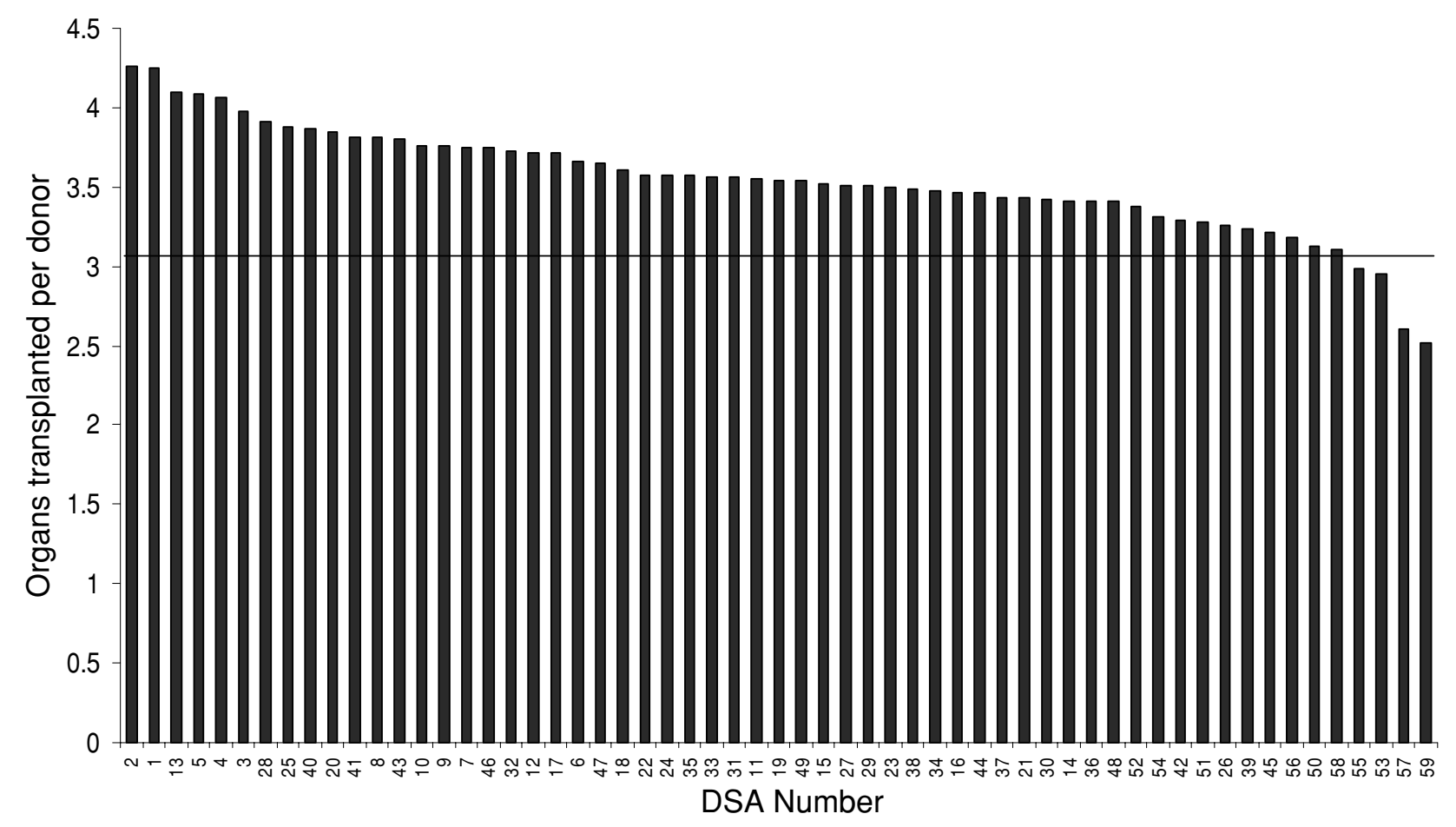

Source: Organ Donation Breakthrough Collaborative.

Figure 4: Organs transplanted per SCD, by DSA, 2003.

for informed consent and designated donation. A registry can be defined in the broadest terms as a database that can be accessed by a recovery agency at any time to get information about whether an individual is listed as willing to be a donor. In early 2006, 42 of 50 states, as well as the District of Columbia, had some form of donor registry. In the United States, $27 \%$ of the population over 16 years old, or 57 million people, are on a donor registry. As large states such as

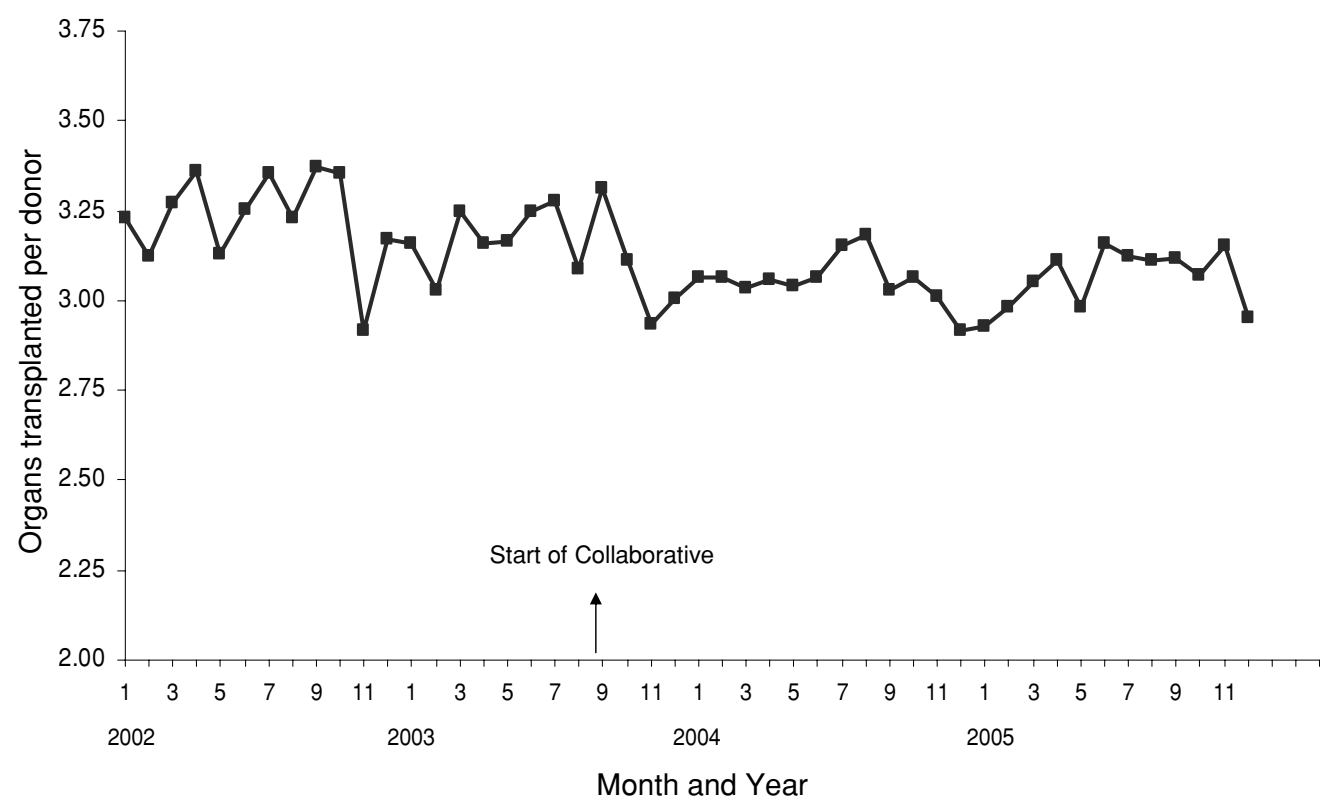

Source: Organ Donation Breakthrough Collaborative.

Figure 5: Organs transplanted per donor pre- and postdonation collaborative (all donors). 
California and Texas implement statewide registries, the national percentage should increase by an additional $5 \%$. Figure 7 demonstrates the status of registries for the entire country. Characteristics of registries vary. Three states offer Internet access only and lack integrated driver's license information. Department of Motor Vehicles (DMV) databases that are not fully integrated exist in 18 states. This hampers the ability to limit gifts or select various options provided by the Uniform Anatomical Gift Act. Access to these databases may be limited by individual DMV policies. There are 21 registries that provide Internet options for registration along with a fully integrated DMV component; these allow the most flexibility. These registries have multiple access points, provide greater ability for data analysis, allow individuals to make changes and allow for the inclusion and effective dissemination of educational materials.

The ongoing efforts to enhance the capabilities and nature of donor registries should contribute to the public's participation in donation and increase the number of organs available for transplantation. Fully integrated models that ensure first-person consent further validate opinion poll results showing that the public wish to donate must be acted upon. Most significantly, as registries evolve and better data are collected, public outreach and education can be more effectively targeted. Historically, public education efforts could never be tied to actual donation rates; improved registries may well provide the answer to this challenge.

\section{Trends in Living Donation}

For the first time in 10 years, there has been a decrease in the number of living donors. In 2005, 6895 living donors were recovered versus 7003 in 2004 . This decrease may be related to multiple factors, including recent negative publicity about living donor safety and a resultant tightening of selection criteria by transplant programs. It could also be related to increased availability of deceased donor organs or to new allocation strategies that allow urgent patients to be transplanted in a more timely fashion than previously.

Living kidney transplant donation: The largest decrease in living donation in 2005 compared with 2004 was seen with kidneys. Demographically, the age of living donors continues to increase. This is reflected by a decrease in donors in the 18-34 year age group and an increase in donors in the 35-49 year, 50-64 year and the $65+$ year age groups. The demographics of kidney donation regarding race, sex and $A B O$ blood type have remained constant. However, the relationship of the donor to the recipient has changed again this year. There is a decrease in sibling-to-sibling donation, from $29 \%$ to $26 \%$. The number of 'unknown' relationships has increased to 214 (3\%) in 2005 compared with $58(2 \%)$ in 2004 . The reason for this increase is unknown, as noted in the 2004 OPTN/SRTR Annual Report (13). The number of unrelated living kidney donors in 2005 was similar to that in 2004. This is true for both spouse (785 (12\%) to $782(12 \%))$ and other unrelated categories (1429 (22\%) to $1446(22 \%))$.

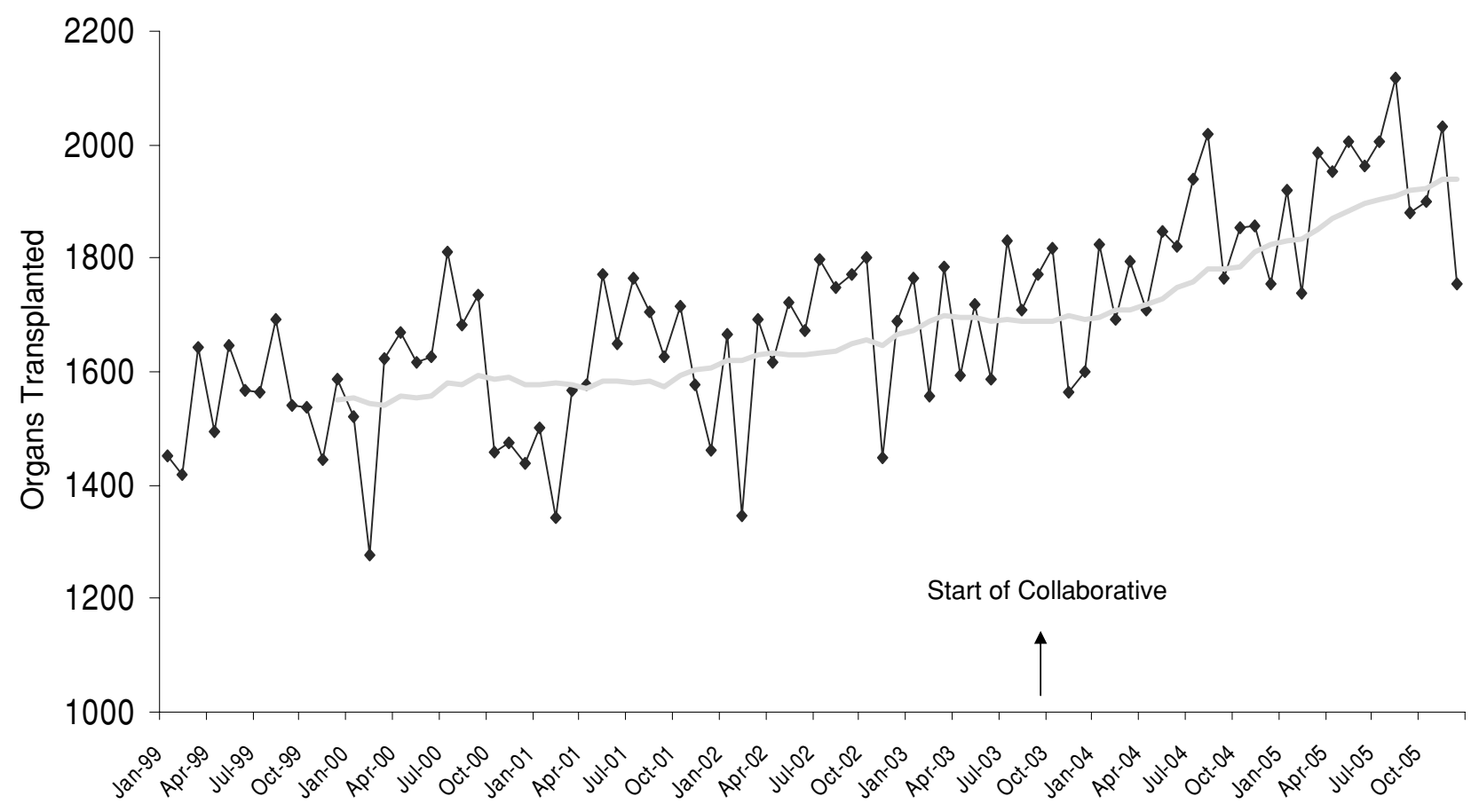

Source: Organ Donation Breakthrough Collaborative.

Figure 6: Organs transplanted per month with 12-month moving average. 


\section{Punch et al.}

Table 6: AOPO recommended definition for 'organ donor potential'

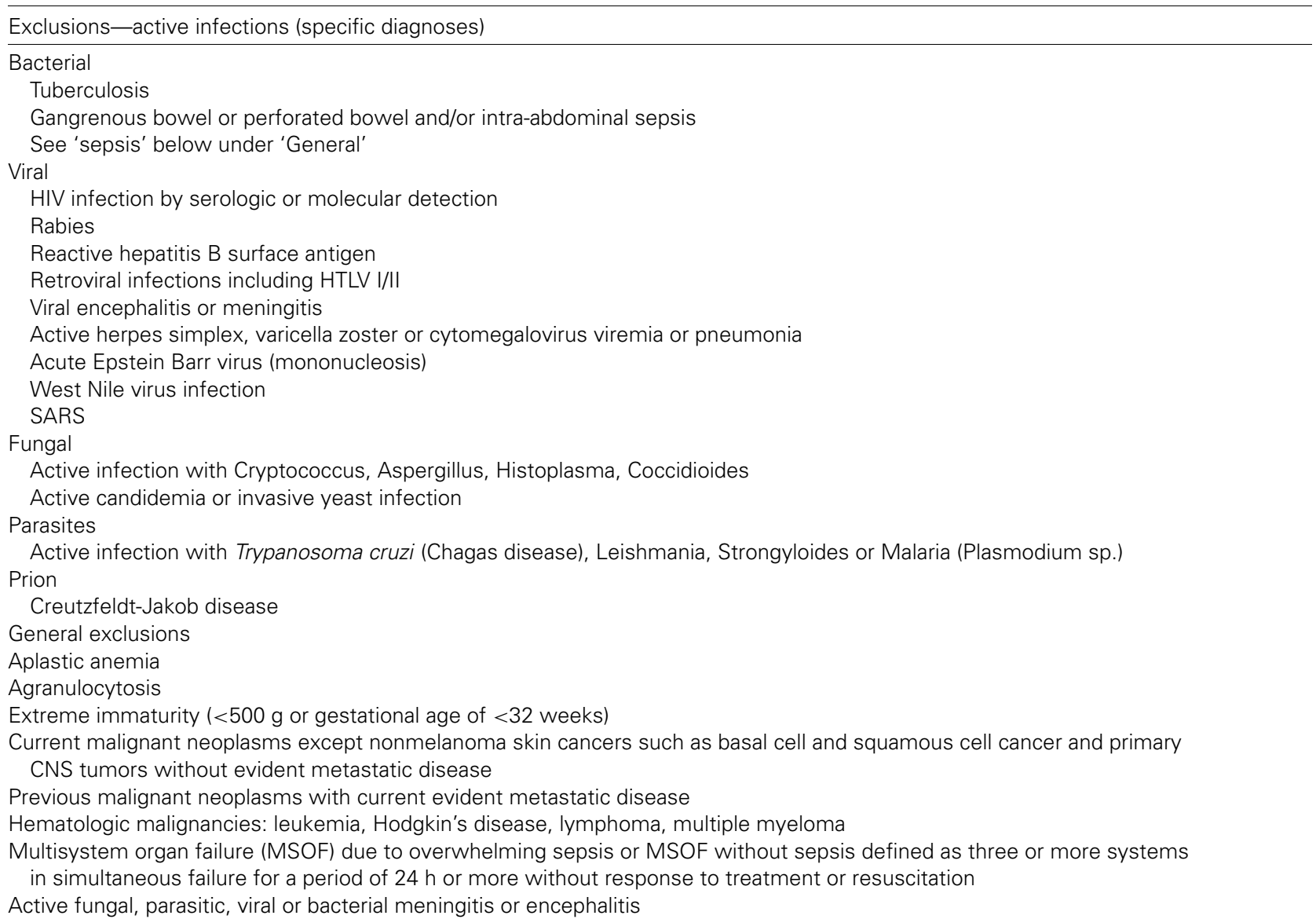

Source: AOPO June 15, 2005 Communication to the Membership.

Inclusions: For reporting purposes, an eligible death for organ donation is defined as the death of a patient 70 years old or younger who ultimately is legally declared brain dead according to hospital policy independent of family decision regarding donation or availability of next-of-kin, independent of medical examiner or coroner involvement in case, and independent of local acceptance criteria or transplant center practice, who exhibits none of the above.

Living pancreas transplant donation: There were no reports of live pancreas donation in 2005.

Living liver donation: The number of living donor liver transplants in the United States has decreased gradually since a high of 519 donors in 2001 but has been relatively stable in the past 4 years. There were 322 donors in 2004 compared with 321 in 2005 . The demographics of living liver donation are changing. The percentage of female donors is increasing, from $44 \%$ in 2003 to 58\% in 2005. Sibling-to-sibling transplants have decreased by $3 \%$, with other unrelated donors decreasing from $23 \%$ in 2004 to $18 \%$ in 2005 . Unknown relationship has sharply increased, from $0.9 \%$ in 2004 to $5 \%$ in 2005 .

Living lung donation: In 2005, there were two living lung donors; both were male, aged between 35 and 49 years. From 2002 to 2004, the number of living lung donors was stable at 25-29 per year. The decrease in 2005 may reflect implementation of the new Lung Allocation Score in May 2005. This system, which rank orders the waiting list based on severity of illness, may have resulted in the allocation of lungs to candidates with short waiting times but high severity of illness. Thus it may have directed lungs to candidates who would have otherwise been considered for living donor transplants.

In recognition of the need to learn more about living donor outcomes, the National Institutes of Health has funded the establishment of living donor follow-up studies.

\section{Summary}

The number of deceased donors has increased by almost $40 \%$ since 1996; most of the increase has occurred since 2000. There are numerous possible factors for this increase: clinical advances and changes in policy now allow 
Source: Intermountain Organ Recovery.

\section{Figure 7: Donor registries across the United States.}

the transplantation of organs that might have been unusable a decade ago; efforts to improve the efficiency and effectiveness of the donation recovery system, most notably the Organ Donation Breakthrough Collaborative, have brought new attention to best practices at hospitals and OPOs nationwide. The role of these factors on the rising number of deceased donors remains untested. The number of living donors dropped slightly from 2004 to 2005, but it is still 82\% greater than it was in 1996.

The essential challenge of providing more organs for transplantation continues. Even as these efforts intensify and the overall number of donors grows, the waiting lists grow even longer. The rate of increase in donation must be maintained as the waiting lists for deceased donor organs continue to grow.

\section{Acknowledgment}

The Scientific Registry of Transplant Recipients is funded by contract number 234-2005-37009C from the Health Resources and Services Administration (HRSA), U.S. Department of Health and Human Services. The views expressed herein are those of the authors and not necessarily those of the U.S. Government. This is a U.S. Government-sponsored work. There are no restrictions on its use.

This study was approved by HRSA's SRTR project officer. HRSA has determined that this study satisfies the criteria for the IRB exemption described in the Public Benefit and Service Program provisions of 45 CFR 46.101(b)(5) and HRSA Circular 03

Note on sources: The articles in this report are based on the reference tables in the 2006 OPTN/SRTR Annual Report, which are not included in this publication. Many relevant data appear in the figures and tables included here; other tables from the Annual Report that serve as the basis for this article include the following: Tables 1.1, 2.1-2.4, 2.7, 2.9$2.18,3.1,3.7,3.13$ and 3.16 . All of these tables may be found online at: http://www.ustransplant.org.

\section{References}

1. Port FK, Bragg JL, Metzger RA et al. Donor characteristics associated with reduced graft survival: an approach to expanding the pool of kidney donors. Transplantation 2002; 74: 1281-1286.

2. Sung RS, Guidinger MK, Lake CD et al. Impact of the expanded criteria donor allocation system on the use of expanded criteria donor kidneys. Transplantation 2005; 79: 12571261.

3. Merion RM, Ashby VB, Wolfe RA et al. Deceased-donor characteristics and the survival benefit of kidney transplantation. JAMA 2005; 294: 2726-2733.

4. Carter JT, Chan S, Roberts JP, Feng S. Expanded criteria donor kidney allocation: marked decrease in cold ischemia and delayed graft function at a single center. Am J Transplant 2005; 5: 27452753.

5. Bernat JL, D'Alessandro AM, Port FK et al. Report of a National Conference on Donation after cardiac death. Am J Transplant 2006; 6: 281-291.

6. Foley DP, Fernandez LA, Leverson $G$ et al. Donation after cardiac death: the University of Wisconsin experience with liver transplantation. Ann Surgery 2005; 242: 724-731.

7. Feng S, Goodrich NP, Bragg-Gresham JL et al. Characteristics associated with liver graft failure: the concept of a donor risk index. Am J Transplant 2006; 6: 783-790.

8. D'Alessandro AM, Fernandez LA, Chin LT et al. Donation after cardiac death: the University of Wisconsin experience. Ann Transplant 2004; 9: 68-71. 


\section{Punch et al.}

9. Marks WH, Wagner D, Pearson TC et al. 2005 SRTR report on the state of transplantation: organ donation and utilization, 1995-2004: entering the collaborative era. Am J Transplant 2006; 6: 11011110.

10. http://www.jointcommission.org/Library/JCAHOnline/j O_06_06.htm (Accessed September 18, 2006).
11. http://newsroom.hrsa.gov/releases/2005/hhs-honors-hospitals. htm (Accessed September 18, 2006).

12. Federal Register. 2006; 71: 30981-31054.

13. Delmonico FL, Sheehy E, Marks WH, Baliga P, McGowan JJ, Magee JC. SRTR report on the state of transplantation: Organ donation and utilization in the United States, 2004. Am J Transplant 2004; 5(4 Pt 2): 862-873. 